\title{
Three-Vector-Based Low Complexity Model Predictive Control for Soft Open Point
}

\author{
Zhengqi Wang $\mathbb{D},{ }^{1}$ Haoyu Zhou $\mathbb{D}^{1},{ }^{1}$ Qunhai Huo $\mathbb{D}^{2},{ }^{2}$ and Sipeng Hao $\mathbb{D}^{1}$ \\ ${ }^{1}$ School of Electric Power Engineering, Nanjing Institute of Technology, Nanjing 211167, China \\ ${ }^{2}$ Institute of Electrical Engineering, Chinese Academy of Sciences, Beijing 100190, China \\ Correspondence should be addressed to Zhengqi Wang; wzqnjit@163.com
}

Received 22 October 2021; Revised 25 November 2021; Accepted 10 December 2021; Published 5 January 2022

Academic Editor: Dazhong Ma

Copyright $\odot 2022$ Zhengqi Wang et al. This is an open access article distributed under the Creative Commons Attribution License, which permits unrestricted use, distribution, and reproduction in any medium, provided the original work is properly cited.

Soft open point (SOP) can improve the flexibility and reliability of power supplies; thus, they are widely used in distribution network systems. Traditional single-vector model predictive control (SV-MPC) can quickly and flexibly control the power and current at both ports of the SOP. However, SV-MPC can only select one voltage vector in a sampling time, producing large current ripples, and power fluctuations. In order to solve the above problems, this paper proposes a three-vector-based low complexity model predictive control (TV-MPC). In the proposed control method, two effective voltage vectors and one zero voltage vector are selected in a sampling time. For the two-port SOP, methods are given to judge the sectors on both sides and select the voltage vectors. Furthermore, the calculation method of the distribution time is proposed as well. Finally, the effectiveness of the proposed method is verified by steady-state and dynamic-state simulation results compared with the SV-MPC.

\section{Introduction}

In recent years, more and more renewable energy sources such as solar energy and wind energy as well as new energy vehicles loads have been connected to the distribution network, which has had a serious impact on the stable and safe operation of the distribution network [1, 2]. Therefore, soft open point (SOP) is used more frequently in distribution networks due to their advantages to flexibly connect feeders of different voltage levels, improve the reliability of power supply, and continuously adjust power [3-5].

The two-port SOP can be regarded as an AC/DC/AC converter composed of voltage source converters (VSCs) [6]. The power exchange at both ports can be realized through corresponding control. In [7], droop control is adopted and proportional-integral (PI) controllers are designed to realize the closed-loop control of the outer power loop and the inner current loop. In [8], the inner current loop of the traditional PI double-loop control is replaced by a sliding mode control, which can reduce the PI controllers. The combination of sliding mode control and feedback linearization control in [9] can meet the requirements of different operating modes. However, control applications will be difficult if a large number of PI coefficients need to be tuned or many controller parameters need to be selected.

Model predictive control (MPC) is widely used in power electronic converters due to its simple implementation and fast dynamic response. According to different control purposes, MPC can be divided into model predictive current control (MPCC) and model predictive power control (MPPC). MPCC and MPPC are used in rectifiers, inverters, modular multilevel converters, induction machines, and permanent-magnet synchronous machines [10-14]. However, the single-vector MPC has only one voltage vector involved in the control during a sampling time, and large current ripples and power fluctuations will be generated, resulting in unsatisfactory control effects. Therefore, how to improve power quality and reduce power fluctuations needs to be considered when MPC is used in converters. In order to improve the control performance, a variety of improved MPC methods have been proposed, such as implementing delay compensation, 
or multistep prediction, improving the cost function, and increasing the number of voltage vectors in a sampling time [15-19]. Among them, increasing the number of voltage vectors is the main way to improve the control performance. In [20], based on the dual-vector MPCC, a method of projecting the current error vector onto the active voltage vector is proposed, which minimizes the cost function and reduces the number of candidate voltage vectors. In [21], different sizes of virtual vectors are defined, the obtained reference voltage vector and the two predicted vectors selected from the virtual vector are used for evaluation, and the virtual vector that minimizes the cost function is used in the next sampling time. This method greatly alleviates the computation burden and avoids the weighting factor in predictive torque control. In [22], a three-vector MPCC is proposed for an openwinding linear permanent-magnet vernier motor. Based on the principle of deadbeat current control, an optimal vector is obtained, and the other two optimal vectors are obtained by cascading cost functions. In addition, the switching frequency is reduced to improve the steady-state performance and suppress the zero-sequence current. In [23], a combination of MPC and direct power control is used for the improved T-type three-level converter, and the switching states are grouped in advance. The capacitor voltage is compared on the DC side to determine the optimal group of the switching state, which eliminates the need for midpoint voltage prediction and cost function calculations, while ensuring the neutral point voltage balance. In [24], the adaptive error correction strategy is applied to both the outer and inner prediction loops from the perspective of model errors, reducing the impact of errors caused by time delay, sampling error, and parameter mismatch on the control performance and effectively improving the output power quality compared to the traditional FCS-MPC.

Both sides of the traditional MPC strategy for the SOP only consider a single switch state control within a sampling time, which results in large current ripples and power fluctuations in the steady state.

The main contribution can be described as follows:

(1) A three-vector-based low complexity MPC scheme is proposed, which reduces the current ripple and power fluctuation by increasing the number of switch states within a sampling time

(2) Methods for judging sectors and selecting vectors are given, which reduce the number of calculations

In Section 2, the mathematical model of the SOP is introduced and the power flow is analyzed. Then, the traditional MPC method for the SOP is introduced, and the control block diagram of the traditional method is given in Section 3. In Section 4, a three-vector-based low complexity MPC method is presented, and methods for judging sectors and selecting vectors at both sides are given. In Section 5, the traditional method and the improved method are compared by simulations, which shows that the proposed method effectively reduces the current ripple and power fluctuation and has good dynamic characteristics. Finally, the article is summarized in Section 6.

\section{Mathematical Model of SOP}

Figure 1 shows the topology of a two-port SOP, where the rectifier side and the inverter side are interconnected by the capacitance of the DC side.

According to Kirchhoff's law, the state equation of the rectifier side and the inverter side in the motionless reference framework can be obtained as

$$
\begin{gathered}
{\left[\begin{array}{c}
V_{a} \\
V_{b} \\
V_{c}
\end{array}\right]=L_{s} \frac{\mathrm{d}}{\mathrm{d} t}\left[\begin{array}{l}
i_{a 1} \\
i_{b 1} \\
i_{c 1}
\end{array}\right]+R_{s}\left[\begin{array}{l}
i_{a 1} \\
i_{b 1} \\
i_{c 1}
\end{array}\right]+\left[\begin{array}{c}
V_{a 1 N} \\
V_{b 1 N} \\
V_{c 1 N}
\end{array}\right],} \\
{\left[\begin{array}{l}
e_{a} \\
e_{b} \\
e_{c}
\end{array}\right]=\left[\begin{array}{l}
V_{a 2 N} \\
V_{b 2 N} \\
V_{c 2 N}
\end{array}\right]-L_{l} \frac{\mathrm{d}}{\mathrm{d} t}\left[\begin{array}{l}
i_{a 2} \\
i_{b 2} \\
i_{c 2}
\end{array}\right]-R_{l}\left[\begin{array}{l}
i_{a 2} \\
i_{b 2} \\
i_{c 2}
\end{array}\right],}
\end{gathered}
$$

where $V_{x}$ and $i_{\mathrm{x} 1}$ are the grid voltage and input current of the rectifier side, respectively; $e_{\mathrm{x}}$ and $i_{\mathrm{x} 2}$ are the load backemf and output current of the inverter side, respectively; the subscripts $x=a, b, \mathrm{c}$ represents the three phases of the SOP; $L_{\mathrm{s}}$ and $R_{\mathrm{s}}$ are the filter inductance and resistance; and $L_{1}$ and $R_{1}$ are the inverter inductance and resistance, respectively.

The switching states of the SOP is defined as

$$
S_{x}=\left\{\begin{array}{l}
1 \\
0
\end{array},\right.
$$

where $S_{\mathrm{x}}=1$ means that the upper switch of phase $x$ is on and the lower switch is off and $S_{\mathrm{x}}=0$ means that the upper switch of phase $x$ is off and the lower switch is on.

Then, introducing the definition of the switching states into (1) and (2), the values of output voltages of each converter can be determined as

$$
\left[\begin{array}{c}
V_{a i N} \\
V_{b i N} \\
V_{c i N}
\end{array}\right]=\frac{u_{d c}}{3}\left[\begin{array}{ccc}
2 & -1 & -1 \\
-1 & 2 & -1 \\
-1 & -1 & 2
\end{array}\right]\left[\begin{array}{l}
S_{a} \\
S_{b} \\
S_{c}
\end{array}\right],
$$

where $\mathrm{u}_{\mathrm{dc}}$ is the capacitor voltage in the DC link; $V_{a i N}, V_{b i N}$, and $V_{c i N}$ are the output voltages of each side in phases $a, b$, and $c$, respectively; and $i=1$ means the rectifier side and $i=0$ means the inverter side.

In the system of the two-port SOP, eight VSC voltage vectors can be chosen, six of which are active voltage vectors $\left(V_{1}, V_{2}, V_{3}, V_{4}, V_{5}, V_{6}\right)$ and two of which are zero vectors $\left(V_{0}, V_{7}\right)$. These eight vectors are shown in Figure 2.

According to the instantaneous reactive power theory, ignoring the line loss and the self-loss of the SOP, the output active power $P$ and reactive power $Q$ of each SOP port can be obtained as 


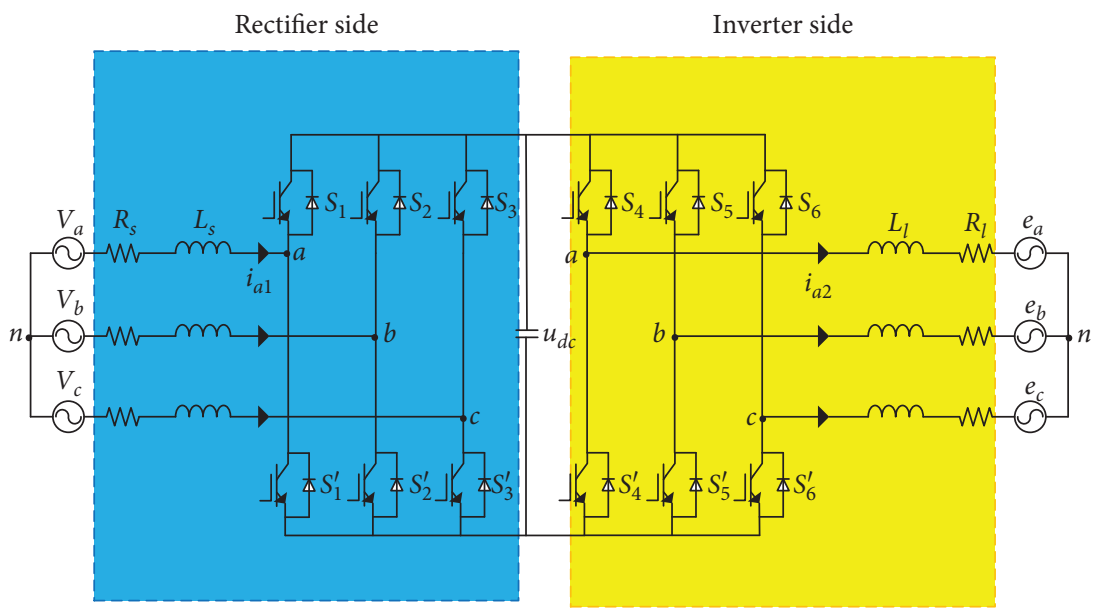

FIgURE 1: Two-port SOP equivalent circuit.

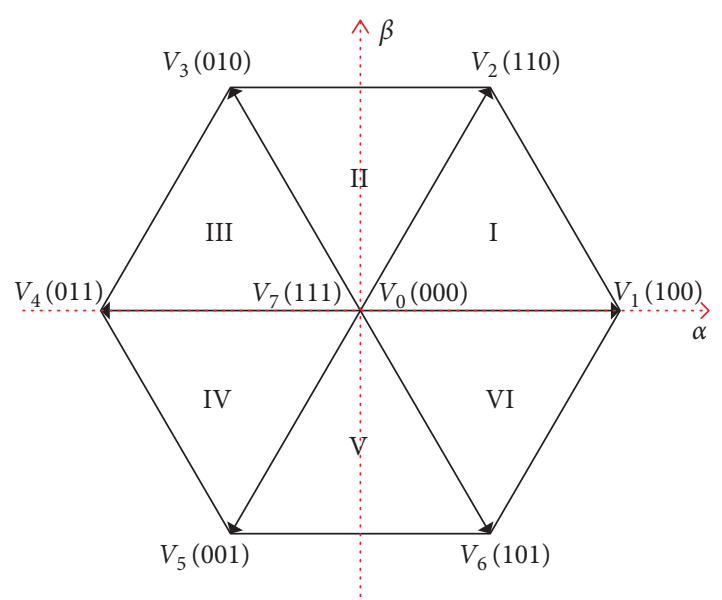

FIGURE 2: Voltage vector in the stationary $\alpha \beta$ reference frame.

$$
\left\{\begin{array}{l}
P_{1}=1.5\left(V_{\alpha} i_{1 \alpha}+V_{\beta} i_{1 \beta}\right) \\
Q_{1}=1.5\left(V_{\beta} i_{1 \alpha}-V_{\alpha} i_{1 \beta}\right) \\
P_{2}=1.5\left(e_{\alpha} i_{2 \alpha}+e_{\beta} i_{2 \beta}\right) \\
Q_{2}=1.5\left(e_{\beta} i_{2 \alpha}-e_{\alpha} i_{2 \beta}\right)
\end{array}\right.
$$

where $P_{1}$ and $Q_{1}$ are the rectifier side active and reactive power, respectively, while $P_{2}$ and $Q_{2}$ are the inverter side active and reactive power, respectively. $V_{\alpha}, V_{\beta}, i_{1 \alpha}$, and $i_{1 \beta}$ are the grid voltage and input current in the stationary $\alpha \beta$ reference frame of the rectifier side, respectively, while $e_{\alpha}, e_{\beta}$, $i_{2 \alpha}$, and $i_{2 \beta}$ are the load back-emfs and output currents in the $\alpha \beta$ reference frame of the inverter side, respectively.

When the SOP operates normally, the output active power of the rectifier side $P_{\mathrm{s}}$ can be calculated by the theorem of conservation of power as

$$
P_{s}=P_{d c}+P_{l},
$$

where $P_{d c}$ is the active power obtained by the DC side capacitor and $P_{1}$ is the active power obtained by the load of the inverter side.

\section{Traditional MPC Analysis}

3.1. Outer-Loop Control Mode Selection. Each port of the SOP can work in different control modes, namely, PQ mode, $\mathrm{U}_{\mathrm{dc}} \mathrm{Q}$ mode, and $\mathrm{U}_{\mathrm{dc}} \mathrm{f}$ mode. One port of the VSC should be needed to control the DC side voltage stability. When the SOP is working normally, its two ports generally work in PQ mode and $\mathrm{U}_{\mathrm{dc}} \mathrm{Q}$ mode. To ensure normal operation and meet load power supply requirements when a feeder fails, the $\mathrm{U}_{\mathrm{dc}} \mathrm{f}$ mode is set and normally used in SOP systems with three or more ports.

In this study, the outer loop of the rectifier side selects $\mathrm{U}_{\mathrm{dc}} \mathrm{Q}$ mode, and the inner loop selects a direct power model predictive control (DPMPC). The outer loop of the inverter side selects a PQ mode, and the inner loop selects the MPCC to reduce the load current error.

3.2. Analysis of DPMPC Method in Rectifier Side. By using the Clark transformation, Equation (1) can be converted to the stationary $\alpha \beta$ reference frame as follows:

$$
L_{s} \frac{\mathrm{d} i_{1 \alpha \beta}}{\mathrm{d} t}=V_{\alpha \beta}-V_{1 N \alpha \beta}-R_{s} i_{1 \alpha \beta},
$$

where $i_{1 \alpha \beta}=\left[\begin{array}{ll}i_{1 \alpha}, & i_{1 \beta}\end{array}\right]^{\mathrm{T}}$ is the input current vector; $V_{\alpha \beta}=\left[V_{\alpha}, V_{\beta}\right]^{\mathrm{T}}$ are the grid voltage vectors of the rectifier side; and $V_{1 N \alpha \beta}=\left[V_{1 N \alpha}, V_{1 N \beta}\right]^{\mathrm{T}}$ are the voltage vectors of the VSC.

Using the forward Euler discretization method to predict the current in the next control period of (7), the predicted current at the $(k+1)$ th instant can be calculated as

$i_{1 \alpha \beta}(k+1)=\left(1-\frac{R_{s} T_{s}}{L_{s}}\right) i_{1 \alpha \beta}(k)+\frac{T_{s}}{L_{s}}\left[V_{\alpha \beta}(k)-V_{1 N \alpha \beta}(k)\right]$,

where $i_{1 \alpha \beta}(k)$ and $V_{\alpha \beta}(k)$ are the input current and grid voltage at the kth instant, respectively; $V_{1 N \alpha \beta}(k)$ is the voltage vector of the VSC in the rectifier side at the kth instant; and $T_{\mathrm{s}}$ is the sampling time. 
In the $\mathrm{U}_{\mathrm{dc}} \mathrm{Q}$ mode, the rectifier side uses a part of the power obtained from the grid to exchange with other ports, and the other part is used to adjust the DC side voltage. The outer loop adopts PI control, and the active power reference value of this port $\underline{P}_{\text {ref }}$ is as follows:

$P_{\text {ref }}=k_{p}\left(u_{d c r e f}-u_{d c}\right)+k_{i} \int\left(u_{d c r e f}-u_{d c}\right) d t+\operatorname{Re}\left\{e \bar{i}_{2 \mathrm{ref}}\right\}$,

where $k_{\mathrm{p}}$ and $k_{\mathrm{i}}$ are the proportional and integral coefficients of PI control, respectively; $\mathrm{u}_{\mathrm{dcref}}$ and $\mathrm{u}_{\mathrm{dc}}$ are the reference voltage and sampling voltage of the DC side, respectively; and $\bar{i}_{2 \text { ref }}$ is the conjugate load reference current of the inverter side. The first two items in (9) calculate the power required to adjust the DC side voltage, and the active power obtained by the load on the inverter side is calculated by the last item.

The voltage vectors and the predicted current vectors can be used to calculate the predicted instantaneous input active power and reactive power as follows:

$\left\{\begin{array}{l}P_{1}(k+1)=1.5\left(V_{\alpha}(k+1) i_{1 \alpha}(k+1)+V_{\beta}(k+1) i_{1 \beta}(k+1)\right) \\ Q_{1}(k+1)=1.5\left(V_{\beta}(k+1) i_{1 \alpha}(k+1)-V_{\alpha}(k+1) i_{1 \beta}(k+1)\right)\end{array}\right.$,

In (10), when the sampling time is small, it can be assumed that $V_{\alpha \beta}(k+1) \approx V_{\alpha \beta}(k)$, but if the sampling time is not small enough to ignore the change in the grid voltage within the sampling time, vector angle compensation can be used to calculate the grid voltage at the $(k+1)$ th instant as follows:

$$
V_{\alpha \beta}(k+1)=V_{\alpha \beta}(k) e^{j \omega T_{s}},
$$

where $\omega$ is the grid voltage pulsation.

Then, Equation (12) can be used as a cost function to evaluate the prediction error generated by each switch, which is described as

$$
f=\left|P_{r e f}-P_{1}(k+1)\right|+\left|Q_{r e f}-Q_{1}(k+1)\right| .
$$

In DPMPC, all eight voltage vectors are substituted into (8) to predict the current at the $(k+1)$ th instant, the predicted powers are substituted into (12) to calculate the error, and the switch with the smallest cost function is selected as the optimal switch and is applied to the SOP rectifier side at the next moment.

3.3. Analysis of MPCC Method in Inverter Side. MPCC is used in the inverter side to compare the predicted load current with the reference load current and select the voltage with the smallest cost function to apply in the next control period.

Transforming (2) to the stationary $\alpha \beta$ reference frame and adopting the forward Euler discretization method, the predicted load current at the $(k+1)$ th instant is as follows:

$i_{2 \alpha \beta}(k+1)=\left(1-\frac{R_{l} T_{s}}{L_{l}}\right) i_{2 \alpha \beta}(k)+\frac{T_{s}}{L_{l}}\left[V_{2 N \alpha \beta}(k)-e_{\alpha \beta}(k)\right]$, where $i_{2 \alpha \beta}(k)=\left[i_{2 \alpha}(k), i_{2 \beta}(k)\right]^{T}$ is the load current at the kth instant; $\mathbf{e}_{\alpha \beta}(k)=\left[e_{\alpha}(\mathrm{k}), e_{\beta}(\mathrm{k})\right]^{\mathrm{T}}$ is the load back-emf in inverter side at the kth instant; and $V_{2 N \alpha \beta}(k)=\left[V_{2 N \alpha}(k)\right.$, $\left.V_{2 N \beta}(k)\right]^{\mathrm{T}}$ is the voltage vector of the VSC in the inverter side at the kth instant.

In order to adjust the load current, a cost function is used to find the best switching state:

$$
g=\left|i_{2 \alpha r e f}-i_{2 \alpha}(k+1)\right|+\left|i_{2 \beta r e f}-i_{2 \beta}(k+1)\right| .
$$

Figure 3 shows the traditional SOP MPC block diagram. At each sampling time, Equations (12) and (14) are used to calculate the cost function values of all switch states on both sides. The switch state that minimizes the cost function is selected to drive the SOP.

\section{Three-Vector-Based Low Complexity MPC Method for SOP}

In the traditional MPC, the VSCs on both sides adopt a singlevector predictive control, the vector selection is very limited, and the voltage vector output by the two ports cannot reach the entire spatial circle track range. As a result, the current ripple content and power fluctuations on both sides become larger. In order to improve the accuracy of control, it is necessary to increase the sampling frequency of the system, thereby increasing the computational burden of the processor. Another problem is that the switching frequency is not fixed. In order to reduce the current ripples and power fluctuations while fixing the switching frequency, a three-vector-based low complexity MPC is used on both sides of the port in this study.

4.1. Cost Function Design. In a three-vector-based MPC, cost functions (12) and (14) can be improved as follows:

$$
\begin{aligned}
& f=\left|P_{\text {ref }}-P_{1}(k+1)\right|^{2}+\left|Q_{\text {ref }}-Q_{1}(k+1)\right|^{2}, \\
& g=\left|i_{2 \alpha \text { ref }}-i_{2 \alpha}(k+1)\right|^{2}+\left|i_{2 \beta \text { ref }}-i_{2 \beta}(k+1)\right|^{2} .
\end{aligned}
$$

In these improved cost functions, the error between the predicted value and the reference value is compared, which is conducive to choosing the best switch value.

Using adjacent effective vectors for vector synthesis, six vector combinations can be obtained: $\left(V_{1}, V_{2}, V_{0,7}\right)$, $\left(V_{2}, V_{3}, V_{0,7}\right), \quad\left(V_{3}, V_{4}, V_{0,7}\right), \quad\left(V_{4}, V_{5}, V_{0,7}\right), \quad\left(V_{5}, V_{6}, V_{0,7}\right)$, and $\left(V_{6}, V_{1}, V_{0,7}\right)$.

In a three-vector-based MPC, since three vectors are synthesized in one sampling time, an additional cost function needs to be designed to comprehensively evaluate the errors of the three vectors in the sampling time. In this study, the weighted root means that the square error is used to design a new cost function:

$$
\omega^{2}=\frac{1}{3}\left(e_{1}^{2} d_{1}^{2}+e_{2}^{2} d_{2}^{2}+e_{0}^{2} d_{0}^{2}\right)
$$

where $e_{1}, e_{2}$, and $e_{0}$ are the cost function values corresponding to the two effective voltage vectors and zero vectors, respectively; and $d_{1}, d_{2}$, and $d_{0}$ are the operating times of the three voltage vectors in the sampling time $T_{\mathrm{s}}$, respectively. 


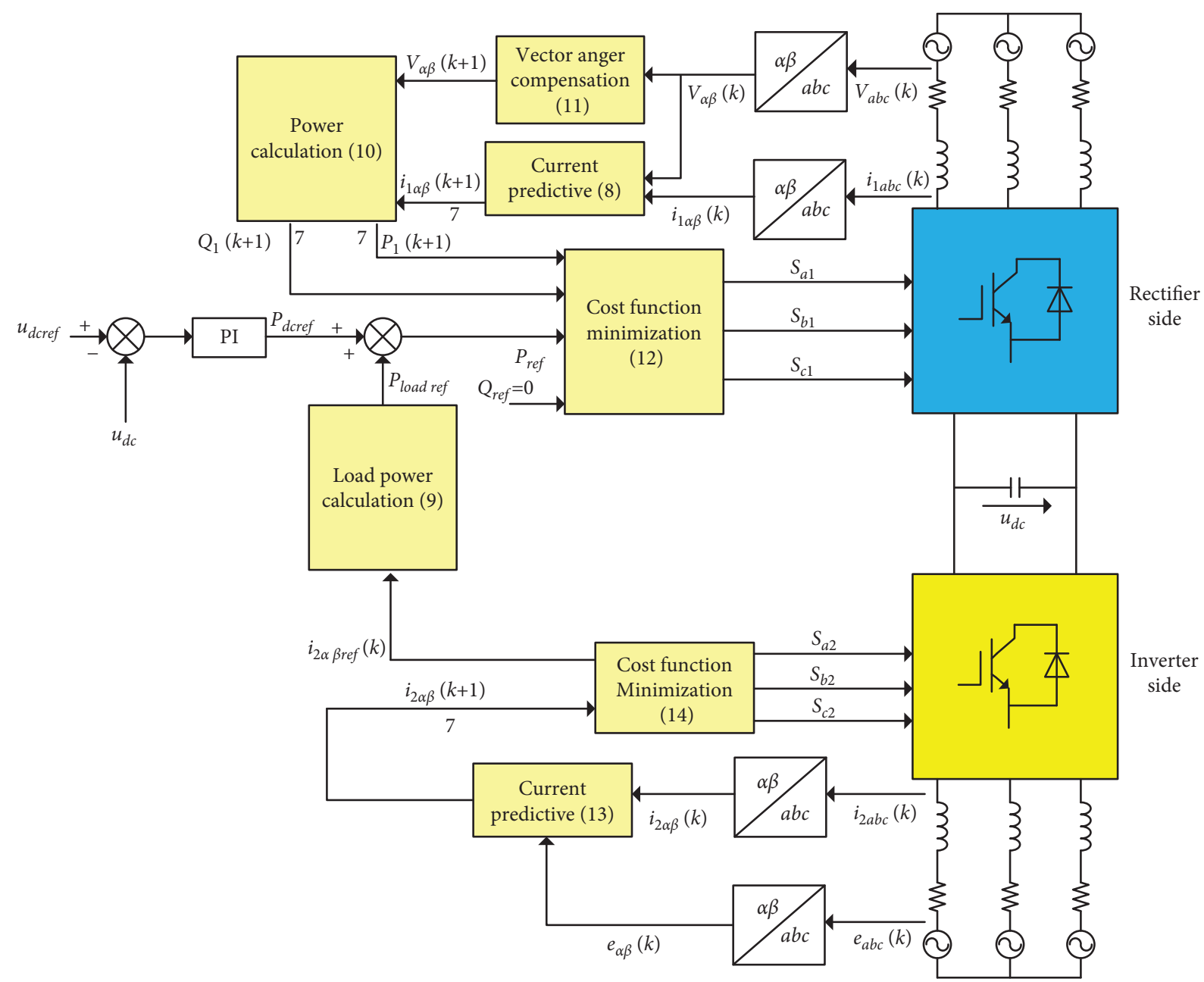

FIGURE 3: Control block diagram of the traditional SOP model predictive control.

Since the sum of squares of errors has been selected as the cost function in (15) and (16), the cost function (17) is written as follows:

$$
G=e_{1} d_{1}^{2}+e_{2} d_{2}^{2}+e_{0} d_{0}^{2}
$$

Taking (18) as the new cost function, the voltage vector time allocation obtained by minimizing the weighted root mean square error is the optimal vector that makes the predicted value of the system closest to the reference value.

4.2. Sector Judgment and Vector Selection. In order to improve the control accuracy and fix the switching frequency, before calculating the duty cycle, it is necessary to judge the reference vector sectors on both sides of the SOP to select the port voltage vector.

On the inverter side, current predictive control is adopted. From the perspective of tracking the reference current, in this study, the inverter side adopts the principle of deadbeat control, which can be derived as follows:

$$
V_{2 N \alpha \beta \mathrm{ref}}=\frac{L_{l}}{T_{s}}\left(i_{2 \alpha \beta \mathrm{ref}}(k)-i_{2 \alpha \beta}(k)\right)+R_{l} i_{2 \alpha \beta}(k)+e_{\alpha \beta}(k),
$$

where $V_{2 N \alpha \beta r e f}$ and $i_{2 \alpha \beta \text { ref }}$ are the reference voltage vectors of the VSC and the reference current, respectively.

After the reference voltage is obtained, the selection of the sector depends on the phase angle $\theta_{\text {ref }}$ of the reference voltage. The phase angle of the reference voltage in the stationary $\alpha \beta$ reference frame can be calculated as follows:

$$
\theta_{\text {ref }}=\arctan \left(V_{2 N \beta \text { ref }} / V_{2 N \alpha \text { ref }}\right) \text {. }
$$

The sectors and vectors selected according to the phase angle $\theta_{\text {ref }}$ are shown in Table 1 . By judging the sectors, the two effective vectors and zero vectors selected on the inverter side are determined, which effectively reduces the amount of calculation.

On the rectifier side, power predictive control is adopted. In order to achieve power tracking, the variables that affect the value of the cost function (15) are analyzed, and the derivative of (5) can be obtained as

$$
\left\{\begin{array}{l}
\frac{d P_{1}}{d t}=\frac{d V_{\alpha}}{d t} i_{1 \alpha}+V_{\alpha} \frac{d i_{1 \alpha}}{d t}+\frac{d V_{\beta}}{d t} i_{1 \beta}+V_{\beta} \frac{d i_{1 \beta}}{d t} \\
\frac{d Q_{1}}{d t}=\frac{d V_{\beta}}{d t} i_{1 \alpha}+V_{\beta} \frac{d i_{1 \alpha}}{d t}-\frac{d V_{\alpha}}{d t} i_{1 \beta}-V_{\alpha} \frac{d i_{1 \beta}}{d t}
\end{array} .\right.
$$


TABLE 1: Selection relationship among sectors, vectors, and phase angles.

\begin{tabular}{lcc}
\hline Phase angle & Sector & Vector selection \\
\hline$\theta_{\text {ref }} \in\left[0^{\circ}, 60^{\circ}\right)$ & I & $V_{1}, V_{2}, V_{7}$ \\
$\theta_{\text {ref }} \in\left[60^{\circ}, 120^{\circ}\right)$ & II & $V_{2}, V_{3}, V_{0}$ \\
$\theta_{\text {ref }} \in\left[120^{\circ}, 180^{\circ}\right)$ & III & $V_{3}, V_{4}, V_{7}$ \\
$\theta_{\text {ref }} \in\left[180^{\circ}, 240^{\circ}\right)$ & IV & $V_{4}, V_{5}, V_{0}$ \\
$\theta_{\text {ref }} \in\left[240^{\circ}, 300^{\circ}\right)$ & V & $V_{5}, V_{6}, V_{7}$ \\
$\theta_{\text {ref }} \in\left[300^{\circ}, 360^{\circ}\right)$ & VI & $V_{6}, V_{1}, V_{0}$ \\
\hline
\end{tabular}

$$
\left\{\begin{array}{l}
\frac{d V_{\alpha}}{d t}=-\omega V_{\beta} \\
\frac{d V_{\beta}}{d t}=\omega V_{\alpha}
\end{array}\right.
$$

Substituting (22) and (7) into (21), (21) can be rewritten

For a three-phase balanced grid voltage, the differential of the grid voltage can be expressed as follows:

$$
\left\{\begin{array}{l}
\frac{d P_{1}}{d t}=V_{\alpha}\left(\frac{V_{\alpha}-V_{1 N \alpha}-R_{s} i_{1 \alpha}}{L_{s}}+\omega i_{1 \beta}\right)+V_{\beta}\left(\frac{V_{\beta}-V_{1 N \beta}-R_{s} i_{1 \beta}}{L_{s}}-\omega i_{1 \alpha}\right) \\
\frac{d Q_{1}}{d t}=V_{\beta}\left(\frac{V_{\alpha}-V_{1 N \alpha}-R_{s} i_{1 \alpha}}{L_{s}}+\omega i_{1 \beta}\right)-V_{\alpha}\left(\frac{V_{\beta}-V_{1 N \beta}-R_{s} i_{1 \beta}}{L_{s}}-\omega i_{1 \alpha}\right)
\end{array} .\right.
$$

Using the forward Euler method to discretize (23), the predictive control of the power in the next sampling period can be obtained as

$$
\left\{\begin{array}{l}
P_{1}(k+1)=P_{1}(k)+f_{p}(k) T_{s} \\
Q_{1}(k+1)=Q_{1}(k)+f_{q}(k) T_{s}
\end{array},\right.
$$

where $f_{\mathrm{p}}$ and $f_{\mathrm{q}}$ are the derivatives of active power $P_{1}$ and reactive power $Q_{1}$, respectively.

For power reference values $P_{1 \text { ref }}$ and $Q_{1 \text { ref, the corre- }}$ sponding voltage vector $V_{1 N r e f}$ is an unknown quantity, which can be obtained from (24) as follows:

$$
\left\{\begin{array}{l}
P_{\text {ref }}=P_{1}(k)+T_{s}\left[V_{\alpha}\left(\frac{V_{\alpha}-V_{1 N \alpha \mathrm{ref}}-R_{s} i_{1 \alpha}}{L_{s}}+\omega i_{1 \beta}\right)+V_{\beta}\left(\frac{V_{\beta}-V_{1 N \beta \mathrm{ref}}-R_{s} i_{1 \beta}}{L_{s}}-\omega i_{1 \alpha}\right)\right] \\
Q_{\text {ref }}=Q_{1}(k)+T_{s}\left[V_{\beta}\left(\frac{V_{\alpha}-V_{1 N \alpha \mathrm{ref}}-R_{s} i_{1 \alpha}}{L_{s}}+\omega i_{1 \beta}\right)-V_{\alpha}\left(\frac{V_{\beta}-V_{1 N \beta \mathrm{ref}}-R_{s} i_{1 \beta}}{L_{s}}-\omega i_{1 \alpha}\right)\right]
\end{array} .\right.
$$

After substituting (25) and (24) into the cost function (15), (15) can be rewritten as

$$
\begin{aligned}
f & =\left|P_{\text {ref }}-P_{1}(k+1)\right|^{2}+\left|Q_{\text {ref }}-Q_{1}(k+1)\right|^{2}, \\
& =\left(\frac{T_{s}}{L_{s}}\right)^{2}\left(V_{\alpha}^{2}+V_{\beta}^{2}\right)\left[\left(V_{1 N \alpha \text { ref }}-V_{1 N \alpha}\right)^{2}+\left(V_{1 N \beta \text { ref }}-V_{1 N \beta}\right)^{2}\right] .
\end{aligned}
$$

where $\left(T_{s} / L_{s}\right)^{2}\left(V_{\alpha}^{2}+V_{\beta}^{2}\right)$ is a constant, so the value of cost function $f$ is only related to $\left(V_{1 N \alpha r e f}-V_{1 N \alpha}\right)$ ${ }^{2}+\left(V_{1 N \beta r e f}-V_{1 N \beta}\right)^{2}$.

Assuming that the voltage reference value $V_{1 \mathrm{Nref}}$ is in sector I, the Euclidean distance between the reference voltage and each effective voltage vector is shown in Figure 4 . The two voltage vectors that minimize $\left(V_{1 \text { Naref }}\right.$ $\left.-V_{1 N \alpha}\right)^{2}+\left(V_{1 N \beta r e f}-V_{1 N \beta}\right)^{2}$ are $V_{1}$ and $V_{2}$. Both $V_{1}$ and $V_{2}$ are in sector I. Therefore, it can be proved that the two effective voltage vectors that minimize the cost function $f$ must be in the same sector as the reference voltage vector $V_{1 N r e f}$, ensuring that the two effective voltage vector combinations with the smallest cost function must be within the six voltage vector combinations listed in Section 4.1.

In the traditional three-vector based MPC, the theory of ergodic optimization is used and all combinations formed by the two active vectors and the zero vector need to be calculated. For the combination of the six active vectors, each side of the SOP needs to calculate 15 times to find the best combinations and action time of each vector at each sampling time. In this paper, the low complexity method proposed above needs only one calculation of the reference prediction voltage on the inverter side, while on the rectifier side, two active voltage vectors with the smallest values of the cost function among the active vectors are proved to be the optimal combination of the active vectors, which requires 


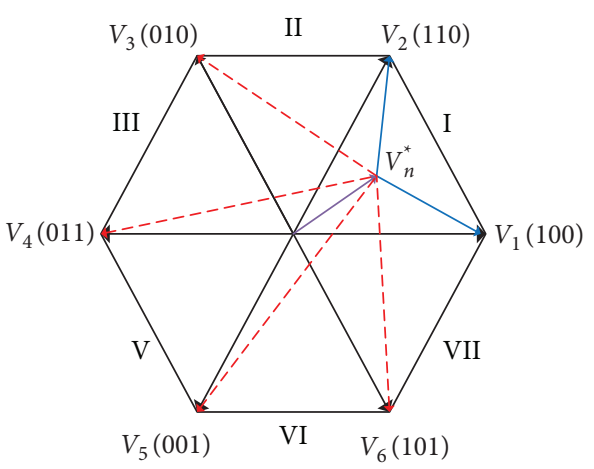

Figure 4: The relative error of each vector and the reference voltage.

only six calculations of cost function and one calculation of action time.

4.3. Calculation of Duty Cycle. In (18), cost function $G$ needs to be minimized to obtain the responsible vector combination; $e_{1}, e_{2}$, and $e_{0}$ can be regarded as known quantities in the cost function; and $d_{1}, d_{2}$, and $d_{0}$ are regarded as unknown quantities. It is equivalent to the problem of finding the extreme value of a multivariate function, and the constraint conditions are as follows:

$$
\left\{\begin{array}{l}
0 \leq d_{1} \leq 1 \\
0 \leq d_{2} \leq 1 \\
0 \leq d_{0} \leq 1 \\
d_{1}+d_{2}+d_{0}=1
\end{array} .\right.
$$

For the extreme value problem of a multivariate function with constraints, the Lagrange multiplier method is used to construct the function as follows:

$$
F\left(d_{1}, d_{2}, d_{0}\right)=e_{1} d_{1}^{2}+e_{2} d_{2}^{2}+e_{0} d_{0}^{2}+\mu\left(d_{1}+d_{2}+d_{0}-1\right)
$$

where $\mu$ is the Lagrange multiplier.

The partial derivatives of the three variables of (28) are, respectively, calculated as 0 , and the calculation can be obtained as follows:

$$
\left\{\begin{array}{l}
d_{1}=\frac{n}{e_{1}} \\
d_{2}=\frac{n}{e_{2}} \\
d_{0}=\frac{n}{e_{0}} \\
n=\frac{1}{e_{1}^{-1}+e_{2}^{-1}+e_{0}^{-1}}
\end{array}\right.
$$

After sector judgment and vector selection, the minimum value of the newly constructed cost function

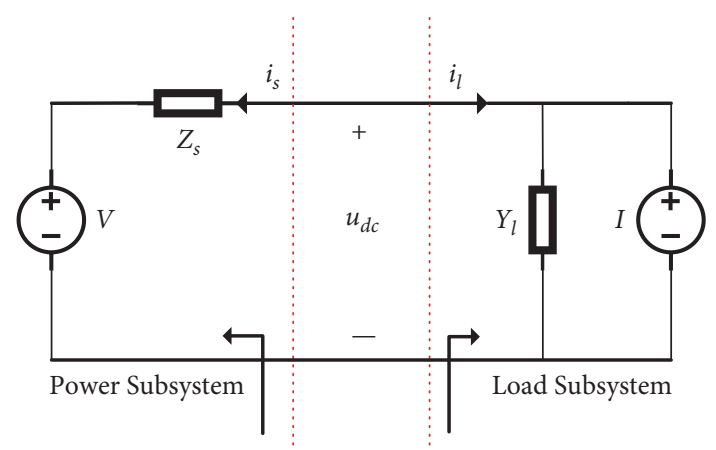

FIGURE 5: Interconnection of two-port SOP.

$F\left(d_{1}, d_{2}, d_{0}\right)$ can be obtained from (29), and the action time of each vector can be calculated, which is applied to the switch control of the next sampling time.

4.4. Stability Analysis. In the two-port SOP, the two-portindependent system can be divided into a power subsystem and a load subsystem depending on the direction of power flow at both sides. As shown in Figure 5, according to the impedance method, the system can only be stable if the subsystem of each port can be stable and the interaction between two ports remains stable at the same time. The system can only be stable when the Nyquist contour of the return-ratio matrix $X$ does not encircle the $(-1, j 0)$ point. By the flow of power, it can be seen that the power positive flow side is the rectifier side, which belongs to the power subsystem, while the negative flow side is the inverter side, which belongs to the load subsystem; thus, the return-ratio matrix $R$ of the two-port SOP can be expressed as follows:

$$
X=Z_{s} Y_{l} \text {. }
$$

The system ensures stability by keeping the eigenvalues of the return-ratio matrix outside the certain forbidden region. In [25], a new forbidden region is proposed as follows:

$$
\left\{\begin{array}{l}
\left|\arg \left(\lambda_{X i}\right)\right| \leq 180^{\circ}-\theta_{P M} \\
\operatorname{Re}\left(\lambda_{X i}\right) \geq-\varepsilon_{G M}
\end{array}\right.
$$

where $\lambda_{X i}$ ( $i=1$ and 2 ) are the eigenvalues of the return-ratio matrix, $\theta_{\mathrm{PM}}$ is the phase margin, and $\varepsilon_{\mathrm{GM}}$ is the gain margin.

As shown in Figure 6, by setting the phase margin and gain margin, $\lambda_{X i}$ can be maintained out of the stability forbidden region.

\section{Summary}

The control block diagram of the three-vector-based MPC for the SOP is shown in Figure 7. Based on the deadbeat principle, the inverter side can calculate the predicted value of the port voltage $V_{2 N r e f}$, the phase angle $\theta_{\text {ref }}$ obtained by $V_{2 N r e f}$ is used to determine the sector where the vector is located, and the sector is used to select the six vector combinations in Section 4.1. The rectifier side selects the two effective vectors with the smallest value function. It has been shown in Section 4.2 that the two vectors with the smallest 


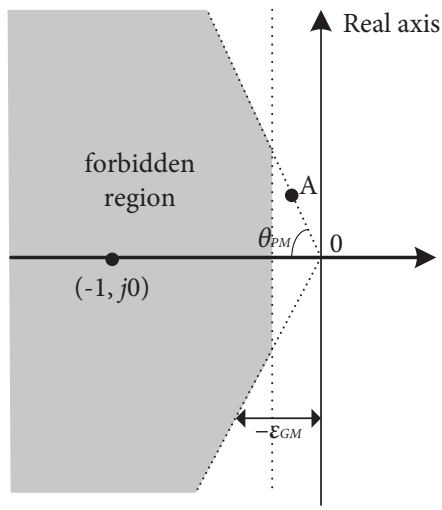

FiguRE 6: Forbidden region of the return-ratio matrix.

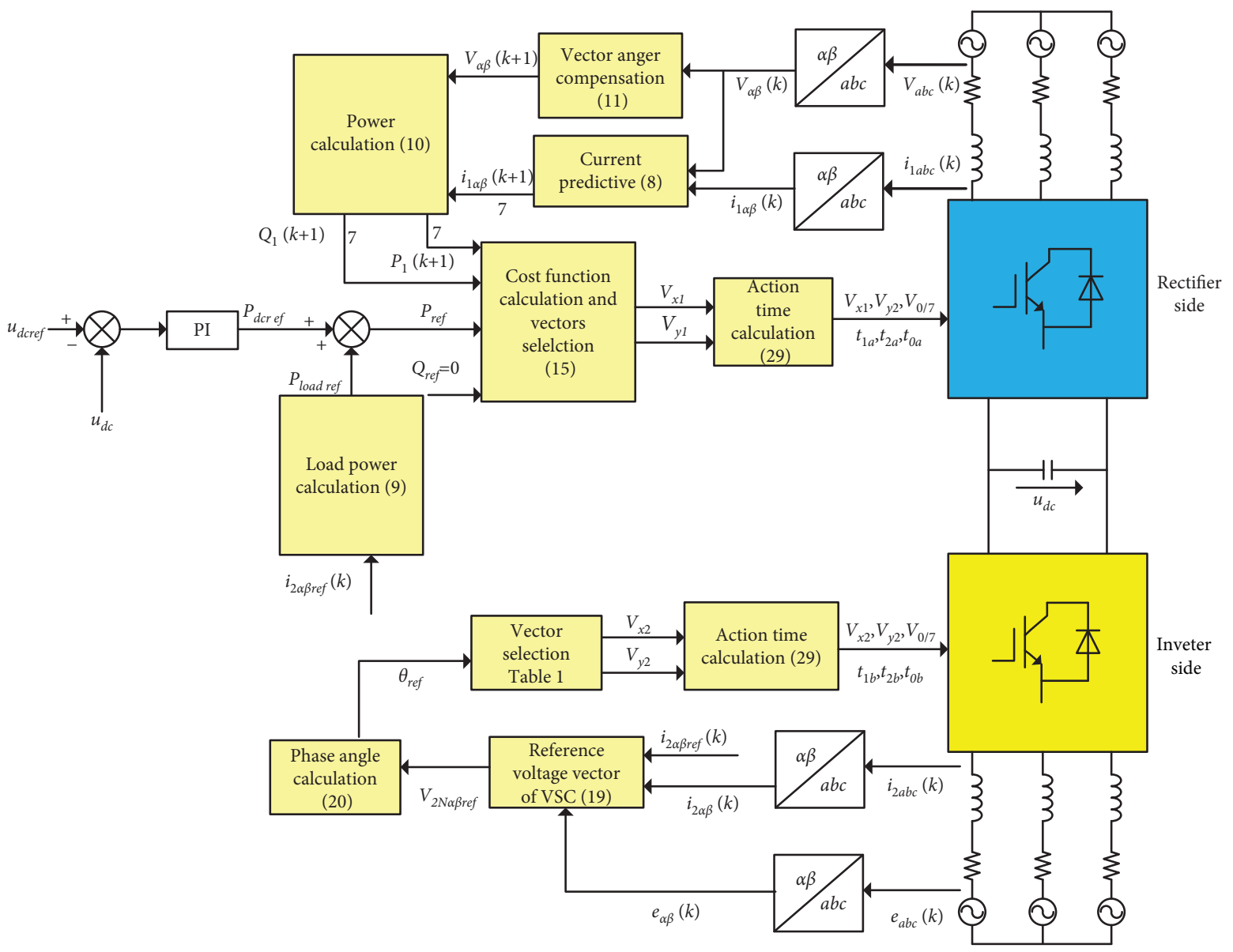

FIGURE 7: Control block diagram of the three-vector-based SOP model predictive control.

value function must be in the same sector, thus ensuring that the selected vector combination must be within the six vector combinations listed in Section 4.1.

Comparing Figure 3 with Figure 7, the three-vectorbased method selects the sector and calculates the duty cycle, differing from the single-vector method where there is only one vector control in the sampling time. As a result, the current ripple and power fluctuations can be reduced, while the switching frequency is fixed.
As shown in Figure 7, only one PI controller is used in the voltage outer loop by the method proposed in this paper. Compared with the seven PI controllers used in the traditional PI loop control [26], the number of PI controllers used is greatly reduced and therefore the complexity of the parameter design is reduced. At the same time, compared with the high accuracy requirements of sliding mode control on system parameters and the high sampling frequency and large output harmonics of hysteresis control, the method 
TABLE 2: Simulation parameters.

\begin{tabular}{lcc}
\hline Descriptions & Parameters & Values \\
\hline Grid voltage & $V_{s}$ & $220 \mathrm{~V}$ \\
Load voltage & $e_{\mathrm{s}}$ & $220 \mathrm{~V}$ \\
Voltage frequency & $f$ & $50 \mathrm{~Hz}$ \\
DC side voltage & $u_{d c}$ & $800 \mathrm{~V}$ \\
DC side capacitor & $C$ & $5000 \mu \mathrm{f}$ \\
Grid resistance & $R_{\mathrm{s}}$ & $0.01 \Omega$ \\
Grid inductance & $L_{\mathrm{s}}$ & $20 \mathrm{mH}$ \\
Load resistance & $R_{\mathrm{l}}$ & $0.01 \Omega$ \\
Load inductance & $L_{\mathrm{l}}$ & $20 \mathrm{mH}$ \\
Sampling time & $T_{\mathrm{s}}$ & $1 \mathrm{e}-4 \mathrm{~s}$ \\
\hline
\end{tabular}
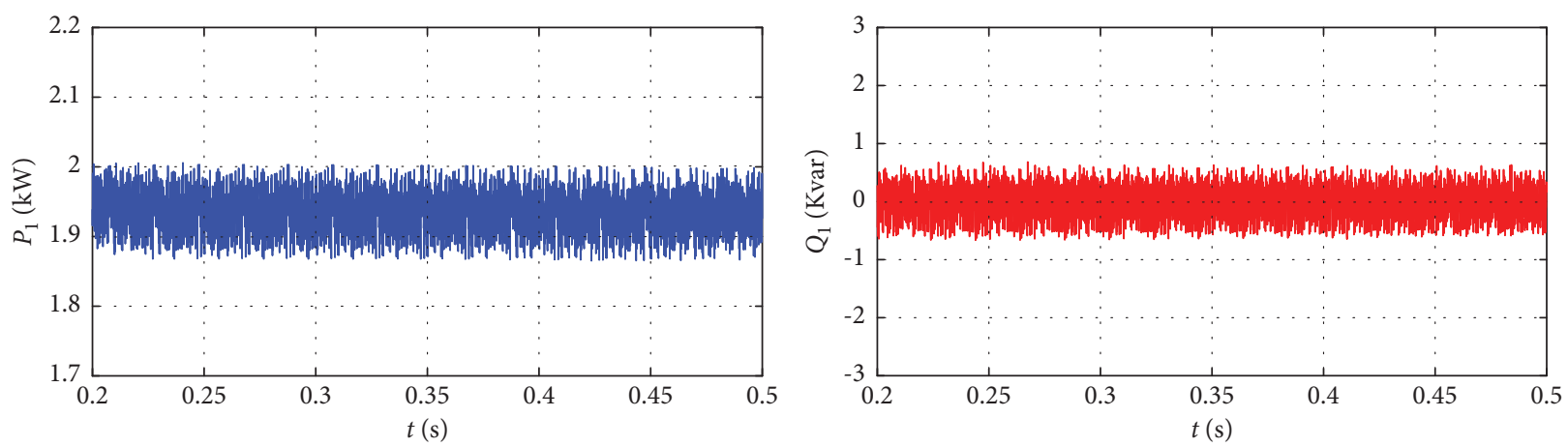

(a)
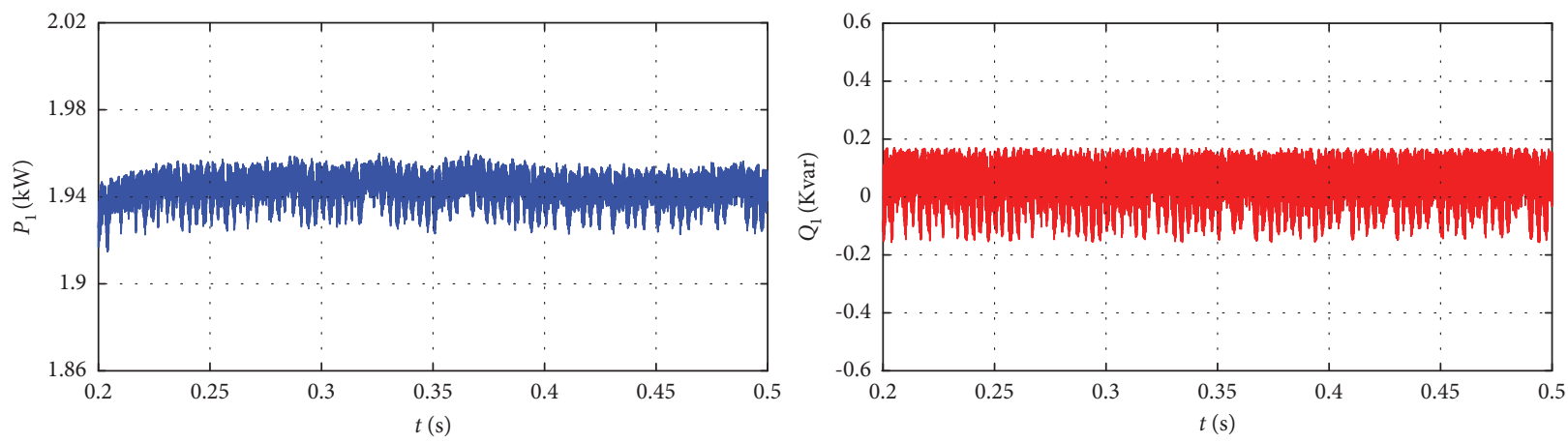

(b)

FIgure 8: Power fluctuations of two methods: (a) SV-MPC; (b) TV-MPC.

proposed in this paper can achieve a simpler control process and operate with better performance.

In the traditional MPC method, a large number of calculations are required at each sampling time, which results in a delay in system control, while the low complexity algorithm requires few calculations at each sampling time. At the same time, using the vector angle compensation for the reference voltage on the rectifier side improves the control accuracy and has a better real-time performance when the sampling time is large.

\section{Simulation Results}

To verify the effectiveness and correctness of the proposed three-vector-based MPC for the SOP, a simulation experiment platform is built in MATLAB/Simulink. The parameters of the SOP are shown in Table 2.
The steady-state simulation of the two methods on the rectifier side is shown in Figures 8 and 9. Figure 8 shows the power fluctuations on the rectifier side of the traditional MPC and three-vector-based MPC when the inverter side reference current is $40 \mathrm{~A}$. The power fluctuations of the two methods obtained by the data analysis are shown in Table 3.

Three-vector-based power prediction effectively reduces the amplitude of power fluctuations compared with singlevector power prediction.

Figure 9 shows the current waveform and frequency spectrum of the rectifier side and the inverter side. The current total harmonic distortion (THD) of the traditional MPC is $2.08 \%$, whereas the current THD of the proposed three-vector-based MPC is $0.91 \%$. Three-vector power prediction control reduces both the current ripple and the power fluctuation. In the rectifier side, both methods effectively track the inverter side reference current. The 

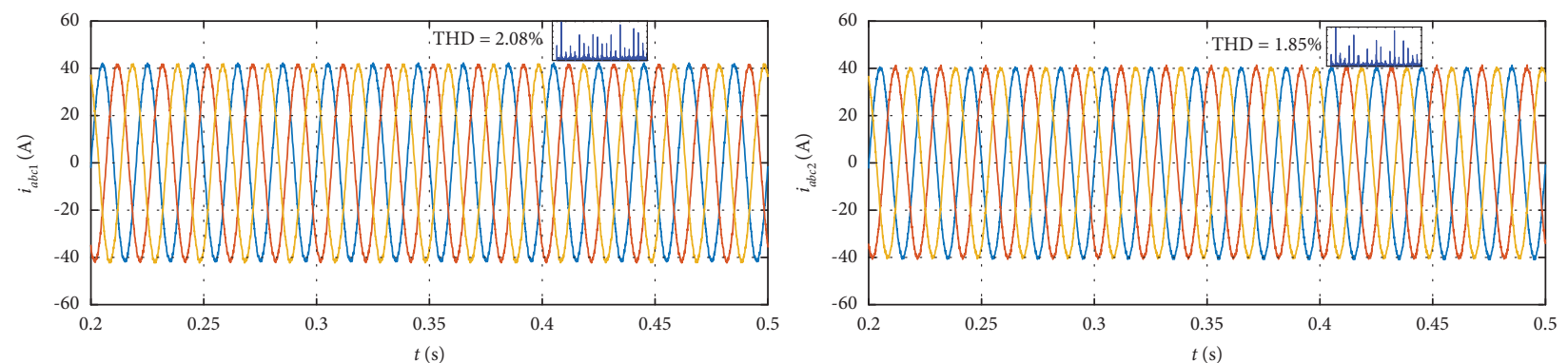

(a)
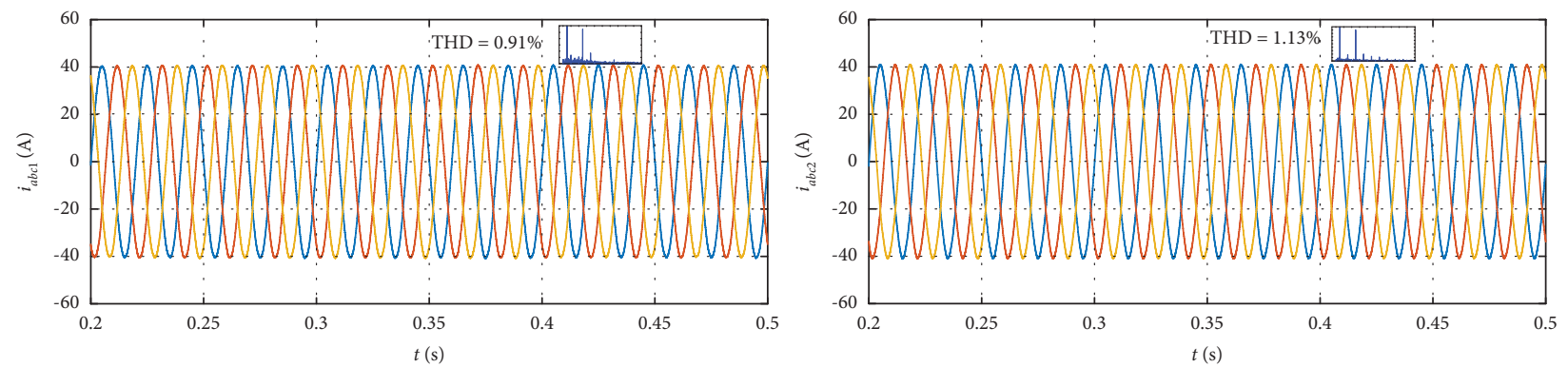

(b)

FIgURE 9: Current waveform and THD of two methods: (a) SV-MPC; (b) TV-MPC.

TABLE 3: Power fluctuations' comparison between two methods.

\begin{tabular}{lcc}
\hline Method & Active power fluctuations (kW) & Reactive power fluctuations (kvar) \\
\hline SV-MPC & 1.408 & 1.349 \\
TV-MPC & 0.463 & 0.328 \\
\hline
\end{tabular}

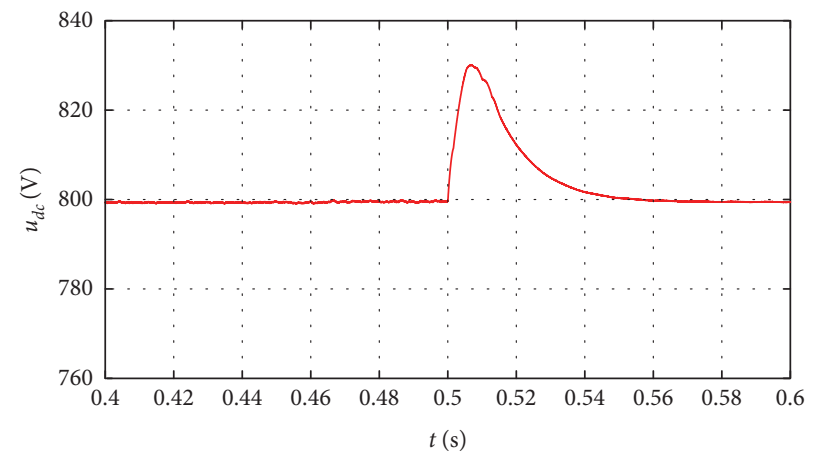

Figure 10: Voltage waveform of DC side under the bidirectional power flow operation.

current THD of the traditional current predictive control is $1.85 \%$, whereas the current THD of the three-vector-based current predictive control is $1.13 \%$, indicating that the proposed method effectively reduces the current ripple.

According to the power and current analysis of two methods on the rectifier and inverter sides in Figures 8 and 9, under steady-state conditions, the three-vector-based method has better stability than the single-vector method. Therefore, the dynamic characteristics of the three-vectorbased method are simulated and analyzed below.

In the actual application of the SOP, it is necessary to meet the bidirectional power flow operation. Figures 10-12 show the dynamic simulation results when the reference current on the inverter side changes suddenly from $40 \mathrm{~A}$ to $-20 \mathrm{~A}$ at $0.5 \mathrm{~s}$. It can be seen from Figure 10 that the voltage change on the DC side is small and then stabilizes to $800 \mathrm{~V}$ again after a short period of fluctuation. The active power and reactive power effectively track the reference power obtained from the inverter side reference current after a short period of fluctuations and meet the conservation of power exchange of two ports in Figure 11. Current waveforms on both sides are shown in Figure 12, and the currents $i_{a b c 1}$ and $i_{a b c 2}$ reach a stable state after small fluctuations. 

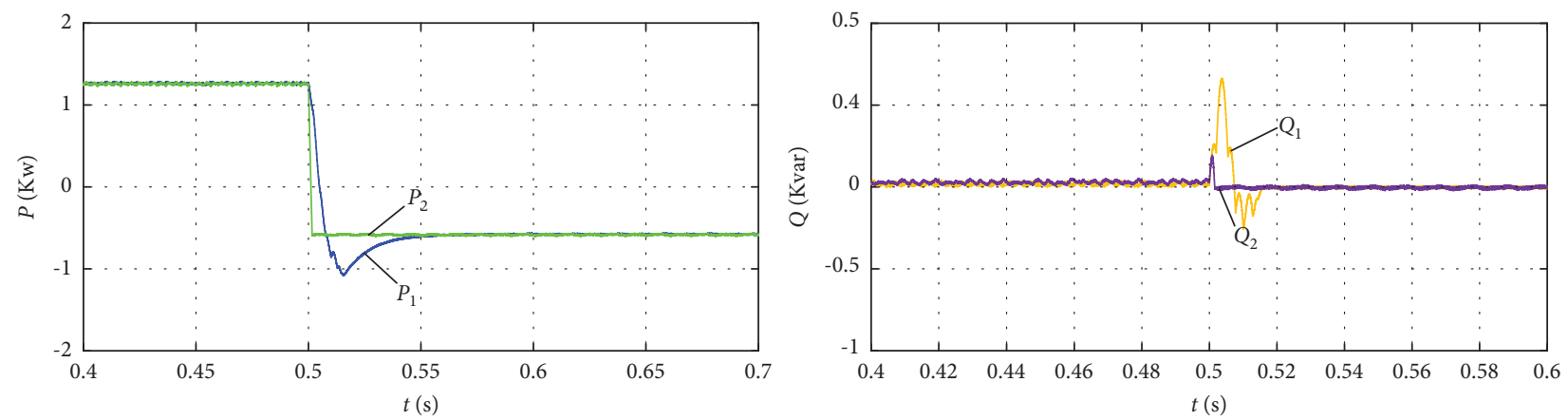

Figure 11: Power fluctuations under the bidirectional power flow operation.
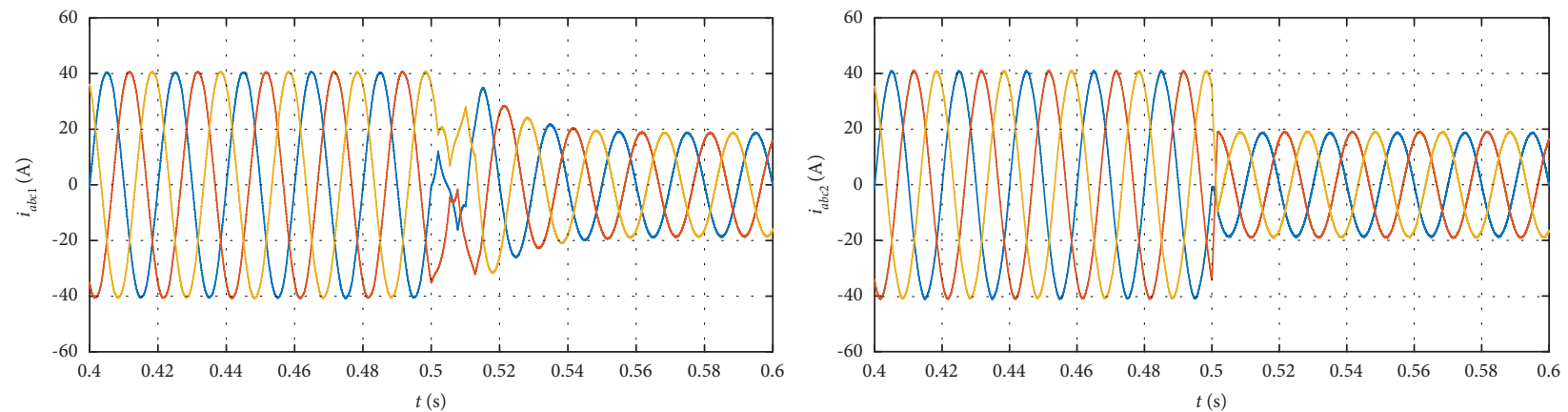

FiguRE 12: Current waveforms under the bidirectional power flow operation.

\section{Conclusion}

This paper proposes a three-vector-based low complexity MPC method for the SOP. First, the power predictive control model on the rectifier side and the current predictive control model on the inverter side are established using the single-vector method. Then, in order to reduce the current ripple content and power fluctuations and fix the switching frequency, two effective voltage vectors and a zero vector are used for modulation to increase the selection range of the voltage vector, and the method of voltage vector selection and action time distribution is proposed. Finally, through the simulation and comparison of the two methods, it is concluded that three-vector-based MPC has a smaller current ripple and a higher power control accuracy, while still maintaining excellent dynamic response characteristics.

However, in the case of sudden changes in the reference current and power, both the DC side voltage and the current of each side have a large fluctuation. The parameter mismatches that can occur in the actual operation are also not taken into account. Therefore, based on the research in this paper, the following research needs to focus on how to combine with other methods to improve the dynamic performance of the system, while ensuring that the system can still operate properly in the case of parameter mismatch.

\section{Data Availability}

The data used to support the findings of this study are included within the article.

\section{Conflicts of Interest}

The authors declare that there are no conflicts of interest regarding the publication of this paper.

\section{Acknowledgments}

This work was supported by the Natural Science Research Project of Jiangsu Higher Education Institution (18KJD470004).

\section{References}

[1] S. Xia, S. Bu, C. Wan, X. Lu, K. W. Chan, and B. Zhou, "A fully distributed hierarchical control framework for coordinated operation of DERs in active distribution power networks," IEEE Transactions on Power Systems, vol. 34, no. 6, pp. 5184-5197, 2019.

[2] M. Liu, P. K. Phanivong, Y. Shi, and D. S. Callaway, "Decentralized charging control of electric vehicles in residential distribution networks," IEEE Transactions on Control Systems Technology, vol. 27, no. 1, pp. 266-281, 2019.

[3] F. Yan, X. Chen, W. Tang, R. Yan, and H. Wu, "Reliability and power supply capability evaluation of active distribution networks with four-terminal soft open points," IET Smart Grid, vol. 3, no. 5, pp. 657-666, 2020.

[4] P. Cong, Z. Hu, W. Tang, C. Lou, and L. Zhang, "Optimal allocation of soft open points in active distribution network with high penetration of renewable energy generations," IET Generation, Transmission \& Distribution, vol. 14, no. 26, pp. 6732-6740, 2020. 
[5] H. Ji, C. Wang, P. Li, F. Ding, and J. Wu, "Robust operation of soft open points in active distribution networks with high penetration of photovoltaic integration," IEEE Transactions on Sustainable Energy, vol. 10, no. 1, pp. 280-289, 2019.

[6] J. M. Bloemink and T. C. Green, "Increasing distributed generation penetration using soft normally-open points," in Proceedings of the IEEE PES General Meeting, pp. 1-8, IEEE, Minneapolis, MN, USA, July 2010.

[7] C. Xu, X. Yuan, Y. Xu, Z. Tan, C. Lin, and M. Chen, "Research on feeder power balancing technology based on SNOP droop control," in Proceedings of the 2019 IEEE 10th International Symposium on Power Electronics for Distributed Generation Systems (PEDG), pp. 192-196, IEEE, Xi'an, China, June 2019.

[8] Y. Zhichun, S. Yu, Y. Fan, L. Yang, S. Lei, and H. Wei, "Study of decentralized coordinated control for soft open point of active distribution networks," in Proceedings of the 2019 IEEE Sustainable Power and Energy Conference (iSPEC), pp. 634640, IEEE, Beijing, China, Nov 2019.

[9] B. Li, Y. Liang, G. Wang, H. Li, and J. Ding, "A control strategy for soft open points based on adaptive voltage droop outerloop control and sliding mode inner-loop control with feedback linearization," International Journal of Electrical Power \& Energy Systems, vol. 122, pp. 1-13, 2020.

[10] D. Zhou, C. Jiang, Z. Quan, and Y. Li, "Vector shifted model predictive power control of three-level neutral-point-clamped rectifiers," IEEE Transactions on Industrial Electronics, vol. 67, no. 9, pp. 7157-7166, 2020.

[11] X. Chen, W. Wu, N. Gao, H. S.-H. Chung, M. Liserre, and F. Blaabjerg, "Finite control set model predictive control for LCL-filtered grid-tied inverter with minimum sensors," IEEE Transactions on Industrial Electronics, vol. 67, no. 12, pp. 9980-9990, 2020.

[12] X. Liu, L. Qiu, Y. Fang, Z. Peng, and D. Wang, "Finite-levelstate model predictive control for sensorless three-phase fourarm modular multilevel converter," IEEE Transactions on Power Electronics, vol. 35, no. 5, pp. 4462-4466, 2020.

[13] W. Xu, D. Dong, J. Zou, and Y. Liu, "Low-complexity multistep model predictive current control for linear induction machines," IEEE Transactions on Power Electronics, vol. 36, no. 7, pp. 8388-8398, 2021.

[14] X. Sun, M. Wu, G. Lei, Y. Guo, and J. Zhu, “An improved model predictive current control for PMSM drives based on current track circle," IEEE Transactions on Industrial Electronics, vol. 68, no. 5, pp. 3782-3793, 2021.

[15] J. Gao, C. Gong, W. Li, and J. Liu, "Novel compensation strategy for calculation delay of finite control set model predictive current control in PMSM," IEEE Transactions on Industrial Electronics, vol. 67, no. 7, pp. 5816-5819, 2020.

[16] O. Gulbudak and M. Gokdag, "Asymmetrical multi-step direct model predictive control of nine-switch inverter for dualoutput mode operation," IEEE Access, vol. 7, pp. 164720164733, 2019.

[17] Y. Luo and C. Liu, "Model predictive control for a six-phase PMSM motor with a reduced-dimension cost function," IEEE Transactions on Industrial Electronics, vol. 67, no. 2, pp. 969-979, 2020.

[18] S. S. Yeoh, T. Yang, L. Tarisciotti, C. I. Hill, S. Bozhko, and P. Zanchetta, "Permanent-magnet machine-based startergenerator system with modulated model predictive control," IEEE Transactions on Transportation Electrification, vol. 3, no. 4, pp. 878-890, 2017.

[19] Y. Zhang, W. Xie, Z. Li, and Y. Zhang, "Low-complexity model predictive power control: double-vector-based approach," IEEE Transactions on Industrial Electronics, vol. 61, no. 11, pp. 5871-5880, 2014.

[20] J. Chen, Y. Qin, A. M. Bozorgi, and M. Farasat, "Low complexity dual-vector model predictive current control for surface-mounted permanent magnet synchronous motor drives," IEEE Journal of Emerging and Selected Topics in Power Electronics, vol. 8, no. 3, pp. 2655-2663, 2020.

[21] Y. Luo and C. Liu, "Elimination of harmonic currents using a reference voltage vector based-model predictive control for a six-phase PMSM motor," IEEE Transactions on Power Electronics, vol. 34, no. 7, pp. 6960-6972, 2019.

[22] D. Xu, W. Zhao, H. Tang, X. Song, and R. Xue, “Three-vectorbased model predictive current control with zero-sequence current suppression for open-winding LPMVM drives," IEEE Transactions on Vehicular Technology, vol. 70, no. 1, pp. 225-236, 2021.

[23] G. Yang, S. Hao, C. Fu, and Z. Chen, "Model predictive direct power control based on improved T-type grid-connected inverter," IEEE Journal of Emerging and Selected Topics in Power Electronics, vol. 7, no. 1, pp. 252-260, 2019.

[24] D. Ma, X. Cao, C. Sun et al., "Dual-predictive control with adaptive error correction strategy for AC microgrids," IEEE Transactions on Power Delivery, p. 1, 2021.

[25] R. Wang, Q. Sun, W. Hu, Y. Li, D. Ma, and P. Wang, "SoCbased droop coefficients stability region analysis of the battery for stand-alone supply systems with constant power loads," IEEE Transactions on Power Electronics, vol. 36, no. 7, pp. 7866-7879, 2021.

[26] S. Li and T. A. Haskew, "Analysis of decoupled d-q vector control in DFIG back-to-back PWM converter," in Proceedings of the 2007 IEEE Power Engineering Society General Meeting, pp. 1-7, IEEE, Tampa, FL, USA, June 2007. 